ARTICLE

DOI: $10.1038 / \mathrm{s} 41467-017-00093-7$

OPEN

\title{
Panoramic-reconstruction temporal imaging for seamless measurements of slowly-evolved femtosecond pulse dynamics
}

Bowen $\mathrm{Li}^{1,2}$, Shu-Wei Huang ${ }^{1}$, Yongnan $\mathrm{Li}^{1,3}$, Chee Wei Wong ${ }^{1} \&$ Kenneth K.Y. Wong (1) ${ }^{2}$

\begin{abstract}
Single-shot real-time characterization of optical waveforms with sub-picosecond resolution is essential for investigating various ultrafast optical dynamics. However, the finite temporal recording length of current techniques hinders comprehensive understanding of many intriguing ultrafast optical phenomena that evolve over a timescale much longer than their fine temporal details. Inspired by the space-time duality and by stitching of multiple microscopic images to achieve a larger field of view in the spatial domain, here a panoramicreconstruction temporal imaging (PARTI) system is devised to scale up the temporal recording length without sacrificing the resolution. As a proof-of-concept demonstration, the PARTI system is applied to study the dynamic waveforms of slowly evolved dissipative Kerr solitons in an ultrahigh-Q microresonator. Two 1.5-ns-long comprehensive evolution portraits are reconstructed with 740 fs resolution and dissipative Kerr soliton transition dynamics, in which a multiplet soliton state evolves into a stable singlet soliton state, are depicted.
\end{abstract}

\footnotetext{
${ }^{1}$ Fang Lu Mesoscopic Optics and Quantum Electronics Laboratory, University of California, Los Angeles, CA 90095, USA. ${ }^{2}$ Department of Electrical and Electronic Engineering, The University of Hong Kong, Pokfulam Road, Hong Kong, 999077, China. ${ }^{3}$ School of Physics and The MOE Key Laboratory of Weak Light Nonlinear Photonics, Nankai University, Tianjin 300072, China. Bowen Li and Shu-Wei Huang contributed equally to this work. Correspondence and requests for materials should be addressed to S.-W.H. (email: swhuang@seas.ucla.edu) or to C.W.W. (email: cheewei.wong@ucla.edu) or to K.K.Y.W. (email: kywong@eee.hku.hk)
} 
T he capability of characterizing arbitrary and non-repetitive optical waveforms with sub-picosecond resolution in a single shot and in real-time is beneficial for different fields, such as advanced optical communication ${ }^{1,2}$, ultrashort pulse generation 3,4 , optical device evaluation ${ }^{5}$ and ultrafast bioimaging $^{6-8}$. Moreover, it has helped to unveil the fascinating ultrafast phenomena in optics, such as the onset of modelocking ${ }^{9}, 10$, soliton explosions ${ }^{11,12}$ and optical rogue waves ${ }^{13-15}$, as well as many other fields ${ }^{16-18}$. Temporal imaging is one of the most promising techniques perceived and developed to meet the need of single-shot real-time waveform characterization $7,8,14,15$, 19-28. On the basis of space-time duality ${ }^{19-21}$, quadratic phase modulation (time lens) and dispersion can be properly combined to significantly enhance the temporal resolution ${ }^{14,15,22-26}$ and a record value of $220 \mathrm{fs}$ has been demonstrated ${ }^{26}$. On the other hand, just like there is always a limitation on the field-of-view in any spatial imaging systems, the single-shot recording length of temporal imaging systems has been hitherto limited to $<300 \mathrm{ps}^{23}$. Owing to this limitation, the time-bandwidth product (TBWP, the ratio between the recording length and the temporal resolution) of the state-of-the-art temporal imaging systems has not exceeded $450^{26}$. Such situation hinders the applications of temporal imaging systems to study many important optical nonlinear dynamics, where not only fine temporal details but also long evolution information are necessary for a comprehensive understanding of the phenomena. For example, studying the dynamics of dissipative Kerr solitons ${ }^{29-31}$ is of particular interest because of their potential applications in low-phase noise photonic oscillators ${ }^{32}, 33$, broadband optical frequency synthesizers $^{34,35}$, miniaturized optical clockwork ${ }^{36}$ and coherent terabit communications ${ }^{37}$. While the soliton generation benefits greatly from the ultrahigh-quality factor $(Q)$ of the microresonator, the ultrahigh $Q$ also renders its formation and transition dynamics slowly evolved at a timescale much longer than the cavity roundtrip time ${ }^{38,39}$, which causes significant challenges in the experimental real-time observation. Similarly, an optical metrology system that combines the feats of fine temporal resolution and long measurement window is also desired in the study of optical turbulence and laminar-turbulent transition in fibre lasers ${ }^{40,41}$, which leads to a better understanding of coherence breakdown in lasers and laser operation in far-from-equilibrium regimes. To capture comprehensive portraits of these processes, as well as many other transient phenomena in nonlinear optical dynamics $^{14,15,42,43}$, a temporal imaging system with a TBWP much greater than 1000 is necessary.

While the most straightforward way to implement a time lens is to use a phase modulator, the TBWP using this approach is fundamentally limited by the maximum achievable modulation depth and is typically $<10^{44-46}$. Alternatively, a time lens can be constructed all-optically through cross-phase modulation ${ }^{47,} 48$. However, similar limitations exist, since large modulation depth requires high pump power, which in turn induces self-phase modulation on the pump pulse and distorts the temporal intensity envelope. Consequently, the reported TBWPs using this approach are only $\sim 20^{47,48}$. Therefore, state-of-the-art temporal imaging systems are mostly implemented through parametric mixing with a linearly chirped pump pulse, where TBWPs up to several hundred have been achieved $7,8,14,15,22-28$. The practical limitation on further improvement of the TBWP in parametric temporal imaging systems originates from the maximum effective pump bandwidth and the maximum pump dispersion ${ }^{20,21}$. While the effective pump bandwidth is restricted by phase-matching condition in parametric conversion, excessive pump dispersion degrades system performance by inducing both large third-orderdispersion (TOD) aberration and undesired propagation loss. Therefore, it is impractical to substantially improve the TBWP of
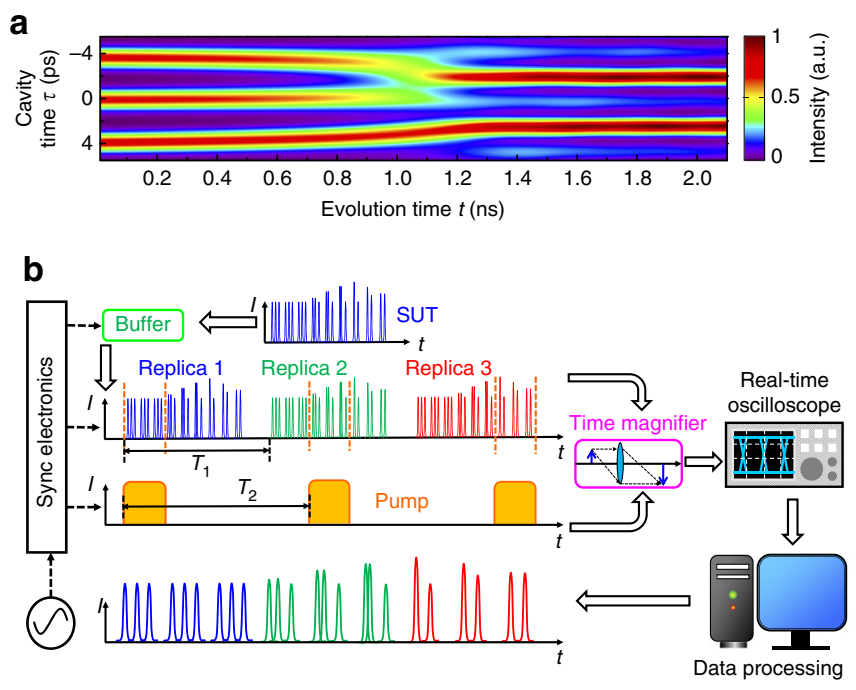

Fig. 1 Working principle of the PARTI system. a Slowly evolved dissipative Kerr soliton dynamics in an ultrahigh- $Q$ microresonator, obtained by numerically solving the Lugiato-Lefever equation. The orders-of-magnitude difference in the timescale between the cavity time and the evolution time poses an experimental challenge to capture the comprehensive picture of the dynamics. $\mathbf{b}$ The schematic representation of the PARTI system. The optical buffer generates multiple replicas (represented by blue, green and red, respectively) of the SUT and the subsequent time magnifier captures different portions of the SUT waveform on each replica. After data processing on the system output, the original long SUT waveform can be reconstructed through waveform stitching

temporal imaging systems under conventional configuration. Meanwhile, limitations on TBWP also exist for other techniques that achieve comparable performance ${ }^{4,}{ }^{49-52}$. Single-shot realtime spectral interferometry ${ }^{52}$ has been adopted to reconstruct the time-domain information, achieving a temporal resolution of $\sim 400 \mathrm{fs}$. However, its temporal recording length is limited by the spectral resolution $(10 \mathrm{pm})$ to $\sim 350 \mathrm{ps}$, which results in a TBWP of 875. Another measurement technique combines spectral slicing of the optical signal with parallel optical homodyne detection using a frequency comb as a reference ${ }^{51}$. Even though a TBWP larger than 320,000 has been demonstrated at a temporal resolution of $\sim 6 \mathrm{ps}$, it is practically challenging to reach the subpicosecond regime. Acknowledging current existing methods, a waveform measurement technique achieving sub-picosecond temporal resolution and long temporal recording length is urgently needed and it will be a powerful tool for studying ultrafast dynamics in different areas.

In order to achieve this goal, we propose and experimentally demonstrate a panoramic-reconstruction temporal imaging (PARTI) system, in analogy with the wisdom of stitching multiple mosaic images to achieve larger-field-of-view in the spatial domain $^{53,54}$. The PARTI system consists of a high-fidelity optical buffer, a low-aberration time magnifier and synchronizationcontrol electronics. Through the PARTI system, different parts of a transient optical dynamic waveform can be characterized sequentially in multiple steps. After signal processing, a magnified panoramic image of the original waveform is reconstructed from multiple mosaic images. A temporal recording length of $1.5 \mathrm{~ns}$ is realized without sacrificing the 740 fs resolution, thus achieving a TBWP of over 2000, about five times larger than the record value previously demonstrated in conventional temporal imaging systems ${ }^{26}$. As a proof-of-concept demonstration, the PARTI system is applied to observe the dissipative Kerr soliton transition dynamics in an ultrahigh- $Q$ microresonator and two distinct 
multiplet-to-singlet dissipative Kerr soliton transition dynamics are observed.

\section{Results}

Principle of operation. Figure 1 shows a simulated example of dissipative Kerr soliton dynamics and describes how the PARTI system captures the slowly evolved process in a single-shot manner. As shown in Fig. 1a, in the governing Lugiato-Lefever formalism $^{55,56}$, the dissipative Kerr soliton dynamics is depicted in a two-dimensional (2D) space spanning by the cavity time $\tau$ and the evolution time $t$. Although the temporal structure of the intra-cavity field is detailed in the $\tau$ dimension at the subpicosecond timescale, the evolution and transition dynamics is portrayed in the $t$ dimension at a much longer nanosecond timescale, which is associated with the cavity photon time of the microresonator. At the beginning of the evolution, the cavity exhibits a triplet soliton state. However, at around $1 \mathrm{~ns}$, the top two solitons start to be attracted to each other and finally merge into a single soliton at $\sim 1.2 \mathrm{~ns}$. The bottom soliton also shifts upwards during the soliton fusion. After $1.6 \mathrm{~ns}$, a stable doublet soliton state is reached, where both solitons exhibit higher intensity owing to the energy conversion inside the microresonator. To comprehensively characterize this soliton-fusion process, a recording length of at least $1 \mathrm{~ns}$ is desired, while a subpicosecond temporal resolution is required to effectively resolve the soliton shape. Therefore, a TBWP larger than 1000 is necessary.

Figure $1 \mathrm{~b}$ shows how the PARTI system overcomes the limitation of TBWP in conventional temporal imaging systems and thus captures the slowly evolved soliton dynamics. The signal under test (SUT) is a pulse train that schematically represents the
2D evolution in Fig. 1a. Since the SUT is transient and nonrepetitive, the concept of sample scanning in the spatial domain cannot be conveniently adopted in temporal imaging systems. To address this problem, a fibre-loop-based optical buffer is integrated with a time magnifier to realize temporal scanning using stroboscopic signal acquisition ${ }^{57,58}$, a technique commonly adopted in sampling oscilloscopes. As shown in Fig. 1b, the optical buffer creates multiple identical replicas of SUT with a constant time interval, which will be subsequently measured by the following time magnifier, thus realizing the temporal scanning on a transient SUT. Using the optical buffer, SUT replicas can be generated with a pre-defined period of $T_{1}$. If the measurement period of time magnifier is $T_{2}$, then in each frame, the time magnifier captures a different section of the long waveform with a step size equal to $\left|T_{1}-T_{2}\right|$. Furthermore, by matching the step size to the recording length of the time magnifier, seamless measurement of a long waveform can be realized. The output of the PARTI system represents the magnified waveform corresponding to different sections of the long SUT and is recorded by a high-speed real-time oscilloscope. After data processing, neighbouring frames of magnified waveform will be stitched together to reconstruct a magnified panoramic image of the original SUT. Therefore, the effective single-shot recording length is scaled by the number of replicas without sacrificing the temporal resolution, thus substantially enhancing the TBWP.

Low-aberration time magnifier. The foundation to construct the PARTI system is a parametric time magnifier with low aberration. The four-wave mixing (FWM) process was chosen as opposed to other parametric processes because it allows high-quality processing of SUT, pump and output simultaneously in the

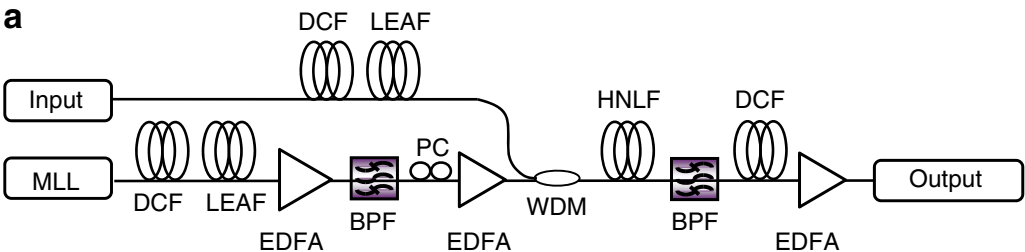

b
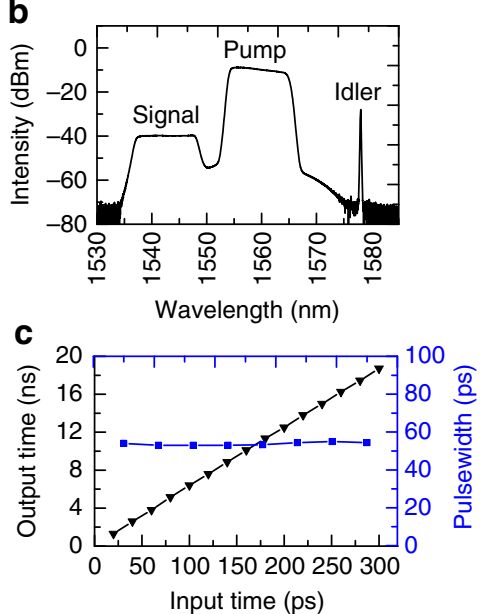

d

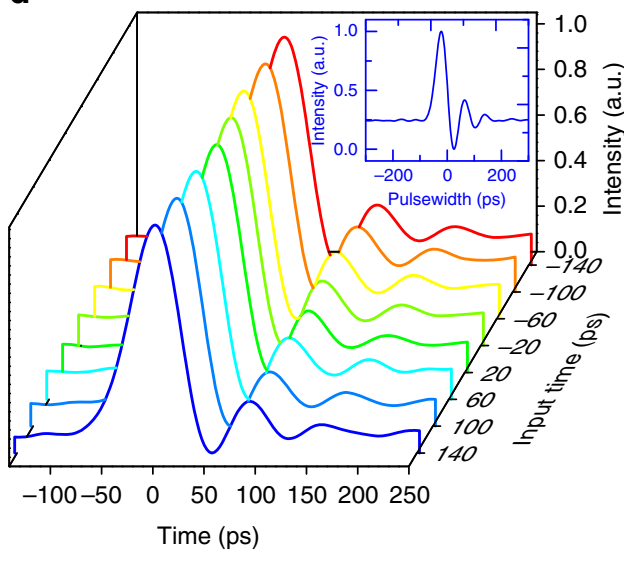

Fig. 2 Schematic representation and performance of the low-aberration time magnifier. a Experimental setup of the low-aberration time magnifier with the parametric time lens implemented through FWM in a $50 \mathrm{~m}$ HNLF. To minimize the third-order-dispersion-induced aberration, both the input and the pump dispersions are provided by proper combination of DCF and LEAF. $\mathbf{b}$ The optical spectrum after the HNLF when measuring a 470 fs pulse. The narrow-band idler is filtered out, dispersed and amplified to become the final output of the time magnifier. c The output time (black, left axis) and pulsewidth (blue, right axis) of the time magnifier as a function of the input time, measuring a temporal magnification ratio of 61.5 and a consistent temporal resolution of $740 \mathrm{fs}$ across the 300 ps recording window. d The normalized output waveforms of the system when the input pulse is temporally shifted across the recording window. The corresponding output waveforms at each input time are labelled by a different colour. (inset) The impulse response of the photodetector. MLL mode-locked laser, EDFA erbium-doped fibre amplifier, BPF band-pass filter, PC polarization controller, WDM wavelength-division multiplexer 
telecommunication band ${ }^{21}$. In addition, since multiple frames of magnified waveform need to be stitched together to obtain the panoramic image, it is critical to ensure a stable impulse response across the recording window of the time magnifier, i.e., a lowaberration FWM time magnifier. For an in-focus time magnifier, the main aberration comes from the TOD in the dispersive path for input and pump ${ }^{59}$. To construct a low-aberration time magnifier with long recording length, the combination of dispersion compensating fibre (DCF) and large effective-area fibre (LEAF) is used to achieve large linear dispersion (fourth and higher-order dispersion neglected). As shown in the experimental setup in Fig. 2a, both the input dispersion and the pump dispersion is provided by combining DCF and LEAF. Since the LEAF has the opposite dispersion slope $\left(0.08 \mathrm{ps} \mathrm{nm}^{-2} \mathrm{~km}^{-1}\right)$ compared to the DCF $\left(-0.598 \mathrm{ps} \mathrm{nm}^{-2} \mathrm{~km}^{-1}\right)$, combining the two types of fibre according to the ratio of their dispersion slope results in linear net dispersion. Moreover, LEAF features in very small dispersion-todispersion-slope ratio $\left(K_{\mathrm{LEAF}}=D / S=45 \mathrm{~nm}\right)$ compared to standard single-mode fibre $(\mathrm{SMF})\left(K_{\mathrm{SMF}}=D / S=275 \mathrm{~nm}\right)$. Therefore, using a LEAF fibre to compensate dispersion slope of DCF sacrifices much less net dispersion compared with using SMF, which facilitates achieving large linear dispersion with moderate insertion loss. In the current system, the SUT is dispersed for 35 $\mathrm{ps}^{2}$ before being combined with the pump through the wavelength-division multiplexer (WDM). In the lower branch of the system, a broad-band mode-locked laser (MLL) goes through a dispersion of $71.2 \mathrm{ps}^{2}$ and is then pre-amplified by a low-noise erbium-doped fibre amplifier. The following band-pass filter selects the spectral component from 1555 to $1565 \mathrm{~nm}$, which is subsequently amplified again to $100 \mathrm{~mW}$ to generate the pump for the time magnifier. The pump and SUT are launched together into the highly nonlinear fibre (HNLF), and the generated idler is filtered out and goes through the output dispersion $\left(2152.5 \mathrm{ps}^{2}\right)$, which is then amplified again to become the final output of the time magnifier. Overall, the system satisfies the imaging condition $^{20}$

$$
\frac{-1}{\Phi_{1}^{\prime \prime}}+\frac{1}{\Phi_{2}^{\prime \prime}}=\frac{1}{\Phi_{f}^{\prime \prime}},
$$

where the $\Phi_{1}^{\prime \prime}\left(35 \mathrm{ps}^{2}\right), \Phi_{2}^{\prime \prime}\left(2152.5 \mathrm{ps}^{2}\right)$ and $\Phi_{f}^{\prime \prime}\left(35.6 \mathrm{ps}^{2}\right)$ are the input output, and focal group-delay dispersions, respectively while the minus sign originates from the phase conjugation during the chosen parametric process. Therefore, the temporal a

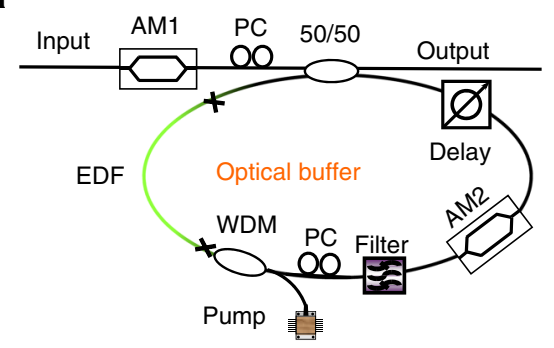

f

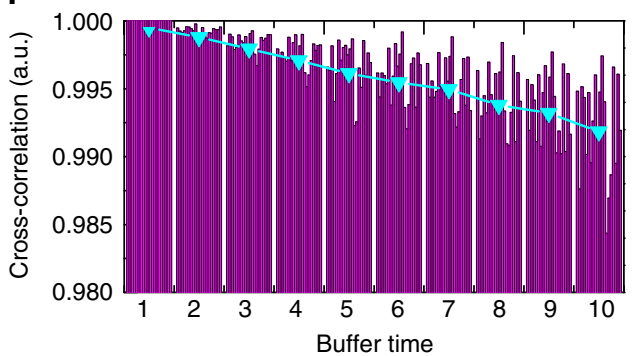

b

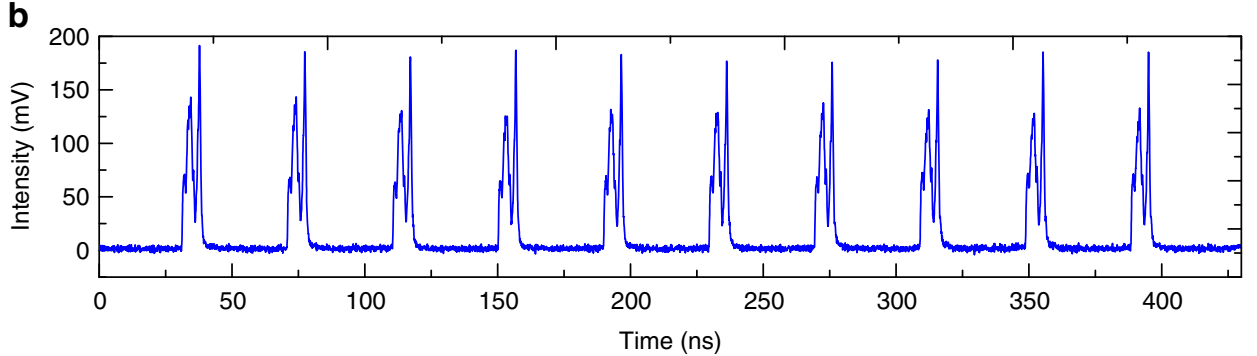

C

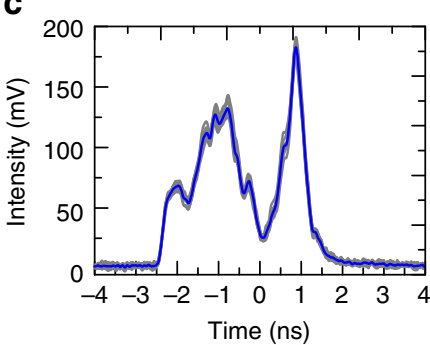

d

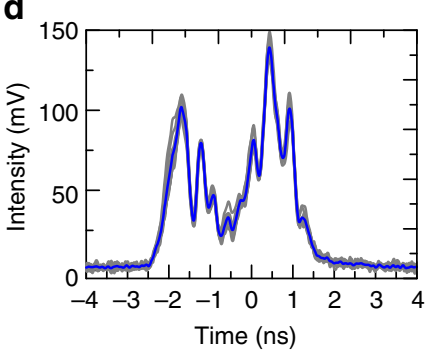

e

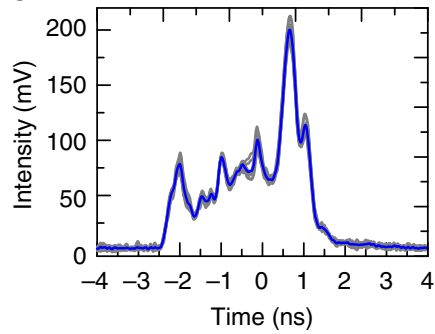

Fig. 3 Schematic representation and performance of the optical buffer. a Experimental setup of the optical buffer, which generates multiple high-fidelity replicas of arbitrary signals under test (SUTs) with fine-tunable period for subsequent stroboscopic signal acquisition. A SUT will be loaded into the buffer through the 50/50 coupler, and one replica will be generated when the SUT is circulated for each cavity round trip. b The output waveform when an arbitrary SUT is optically buffered for 10 times. c-e Three examples of buffering performance using distinct SUTs. Ten replicas are overlapping together (grey curve) and compared to their average (blue). f Quantitative characterization of the buffering performance using 20 different SUTs. Each column set in purple represents the cross-correlation coefficient between the replica after different times of buffering and the original waveform (first replica). The average values among 20 examples are shown by blue triangles, showing a fidelity loss of less than $1 \%$ after ten times of buffering. AM amplitude modulator, EDF erbium-doped fibre, PC polarization controller, WDM wavelength-division multiplexer 
magnification ratio is

$$
M=\frac{\Phi_{2}^{\prime \prime}}{\Phi_{1}^{\prime \prime}}=61.5
$$

To characterize the performance of the time magnifier, a femtosecond pulse with $10 \mathrm{~nm}$ bandwidth and centre wavelength of $1543 \mathrm{~nm}$ is used as input of the system. As shown in Fig. 2b, in the FWM spectrum after the HNLF, a narrow-band idler is generated, which is then filtered out and becomes the temporally magnified signal after output dispersion. The femtosecond pulse is shifted temporally across 300 ps input window (input time scanning) and the corresponding output waveform is recorded. As shown in Fig. 2c, the output time is linearly proportional to the input time with a slope of 61.5. Moreover, the output pulse width during input time scanning is stable at $54 \mathrm{ps.} \mathrm{The}$ corresponding output pulse shape (intensity normalized individually) is also almost identical (Fig. 2d), which indicates very small aberration from TOD. This feature is most critical for implementing the temporal scanning microscope, as ideally the overlapping areas should be identical in neighbouring measurement frames so as to be clearly identified for image stitching. The small fluctuating tail of the waveform results from the impulse response of the photodetector, which is shown in the inset. On the basis of the results above, the average measured pulse width is $54 \mathrm{ps} / 61.5=878 \mathrm{fs}$. Since the real pulse width of the input signal is measured to be $470 \mathrm{fs}$ through auto-correlation, the deconvolved impulse response or the temporal resolution of the time magnifier is calculated to be $\sqrt{878^{2}-470^{2}}=740 \mathrm{fs}$. Therefore, the low-aberration time magnifier achieves a large TBWP of $300 \mathrm{ps} / 740 \mathrm{fs}=405.4$ enabled by the large linear dispersion links in the system, which is comparable to the largest TBWP previously demonstrated in temporal imaging systems ${ }^{26}$.

Optical buffer and timed replication. To generate multiple replicas of SUT for subsequent stroboscopic signal acquisition (discussed in the next section), a fibre-loop-based optical buffer is designed and the experimental setup is shown in Fig. 3a. During operation, a section of waveform will be carved out by amplitude modulator 1 (AM1) and loaded into the buffer through a 50/50 coupler. After each circulation inside the fibre-loop cavity, 50\% of the buffered waveform is coupled out as a replica, while the other $50 \%$ is circulated for the next round. The total cavity length is designed to be around $8.2 \mathrm{~m}$ and the cavity period can be finetuned from 39.7 to $40 \mathrm{~ns}$ using the optical delay-line in order to match the frame rate of the time magnifier. AM2 functions as a switch by controlling the intra-cavity loss. The switch is turned on only when the SUT passes the AM2 and therefore, the AM2 controls the number of replicas generated from the buffer. More importantly, the periodic switching of AM2 prevents the selflasing operation of the optical buffer, which substantially suppresses the amplification noise during the buffering. In addition, a WDM filter with a passband from 1537 to $1547 \mathrm{~nm}$ further minimizes the buffering noise. A $2 \mathrm{~m}$ erbium-doped fibre (EDF) pumped by $980 \mathrm{~nm}$ laser diode provides a maximum gain of $\sim 20$ $\mathrm{dB}$ to compensate the total cavity loss $(\approx 12 \mathrm{~dB})$. To minimize the dispersion distortion, $0.5 \mathrm{~m}$ DCF is added to the cavity and the net dispersion of the buffer is measured to be $\sim 6.12 \times 10^{-3} \mathrm{ps}^{2}$ (see Supplementary Note 1 for details), which corresponds to the dispersion of only $0.28 \mathrm{~m} \mathrm{SMF}$. For a $740 \mathrm{fs}$ optical pulse (equal to the resolution of the time magnifier), such residual dispersion will only result in $<5 \%$ pulse shape distortion after ten roundtrips. Therefore, the influence of residual net dispersion is small enough to be neglected. Finally, by optimizing the polarization controllers
(PC) both outside and inside the cavity, the buffer generates highfidelity replicas of the input waveform.

To visualize the performance of the buffering, arbitrary waveforms (see Supplementary Note 2 for details) generated from an ultrahigh-Q microresonator are used as SUT and launched into the optical buffer to generate ten replicas. All the SUT have the duration of $\approx 5 \mathrm{~ns}$ and spectral bandwidth of $\approx 10$ $\mathrm{nm}$, but the waveforms shapes are distinct from each other. Figure $3 \mathrm{~b}$ shows the output waveform of ten replicas for a certain SUT after the buffering. The shape as well as the intensity of the SUT are well preserved during buffering. In Fig. $3 \mathrm{c}$, the ten replicas in Fig. 3b are overlapping together (grey curves) and are compared to the averaged waveform (blue curve). It is obvious that the optical buffer can generate high-fidelity replicas, which only exhibit small fluctuations $(<10 \%)$ during each buffering compared to the averaged reference. Figure 3d,e show similar performance for two more arbitrary examples of SUT. To quantitatively evaluate the buffering fidelity, a total of 20 different SUTs are tested. As shown in Fig. 3f, for each SUT, the crosscorrelation coefficient between different replicas and the first replica (original waveform) are calculated and represented by purple columns, while the blue triangles shows the average value of the 20 SUTs. The first column set represents the crosscorrelation coefficient of the first replica with itself (i.e., autocorrelation) and therefore the value equals 1 for all 20 SUTs. After the first buffering time, the coefficients start to decrease gradually with each buffering, which indicates larger and larger deviations from the original waveforms owing to the buffering distortions. However, even for the tenth replica, the average cross-correlation coefficient is still larger than 0.99 . Therefore, the optical buffer is able to generate high-fidelity replicas for arbitrary temporal waveforms. As the dispersion broadening is far below the temporal resolution of direct measurement $(\approx 18 \mathrm{GHz}$ bandwidth), the majority of the deviation is attributed to the amplification of noise and gain narrowing effect in the buffer and thus reducing the cavity loss and inclusion of gain equalizers can further improve the quality of the optical buffer, if necessary.

Stroboscopic signal acquisition and the PARTI system. Equipped with the low-aberration time magnifier and highfidelity optical buffer, the PARTI system is implement based on the concept of stroboscopic signal acquisition ${ }^{57,58}$. The basic idea has already been illustrated in Fig. 1. By inducing a period difference between the buffered replicas and the pump pulses, the time magnifier captures a different section of SUT consecutively on each replica. In this way, a long SUT can be fully scanned in multiple steps and the complete waveform can be reconstructed from the magnified waveform of each stroboscopic acquisition. The experimental detail of implementation is shown in Fig. 4a. To emphasize the key components for stroboscopic acquisition, the synchronization electronics are highlighted, while the optical buffer and the time magnifier are simplified and slightly shadowed. The key electronics can be divided into the following three groups. First of all, a repetition-rate-stabilized femtosecond fibre MLL and a $1.2 \mathrm{GHz}$ photodetector together generate a 250 $\mathrm{MHz}$ electrical clock signal, which serves as the time base of the whole system. Secondly, an arbitrary waveform generator (AWG), and a delay generator create electrical patterns that control the stroboscopic acquisition. Finally, the three AMs convert the electrical patterns to the optical domain, which control the SUT loading (AM1), optical buffer switching (AM2) and time-magnifier-pump generation (AM3), respectively.

The detailed timing chart of the system is shown in Fig. 4b. As indicated by the vertical blue dashed line, the whole system is operated with a frame rate of $2 \mathrm{MHz}$. In every $500 \mathrm{~ns}$, the AM1 
a

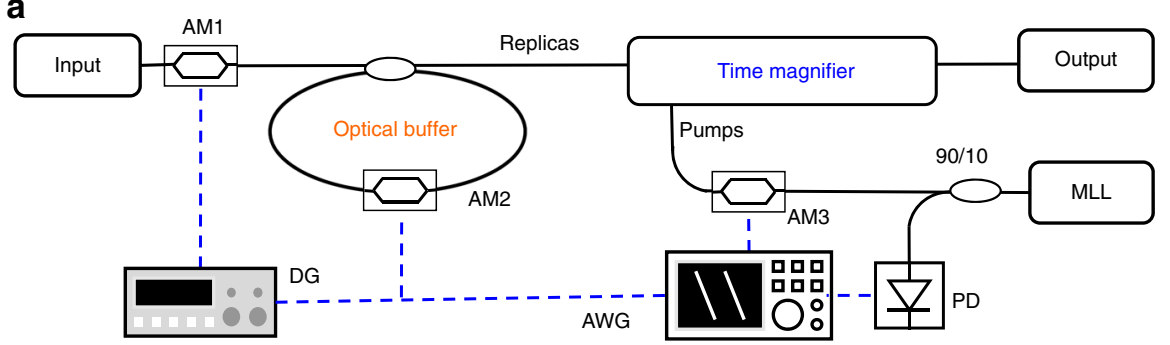

b

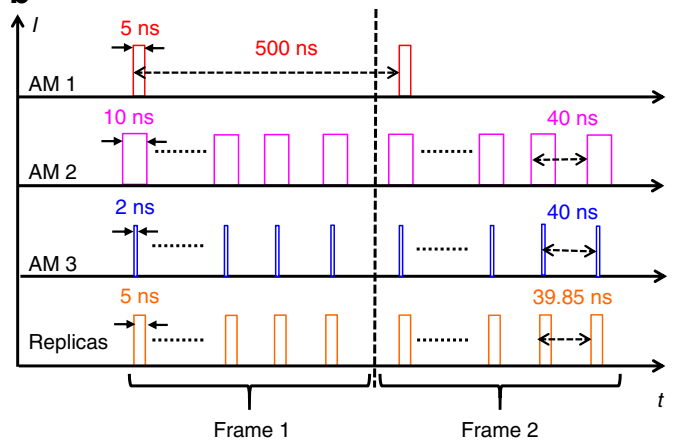

C

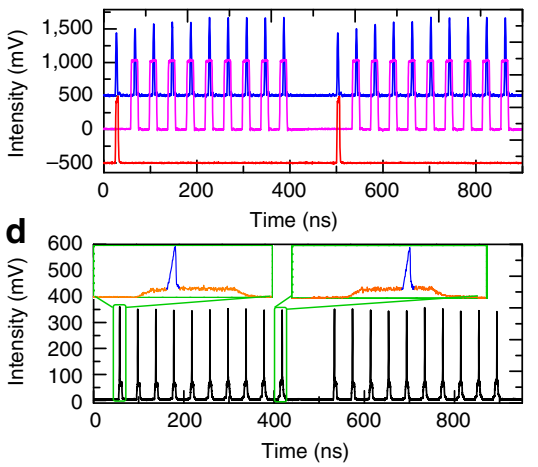

Fig. 4 Implementation of stroboscopic signal acquisition. a Simplified experimental setup of the PARTI system, emphasizing key components for electronic synchronization. A repetition-rate-stabilized mode-locked laser (MLL) and a photodetector (PD) together generate the clock signal for the whole system. An AWG and a delay generator (DG) provide the electrical driving patterns based on the clock signal. Finally the three amplitude modulators (AMs) convert the electrical driving patterns to the optical domain, which control the signal-under-test (SUT) loading (AM1), optical-buffer switching (AM2) and time-magnifier-pump generation (AM3), respectively. b Detailed timing chart of the system with a frame rate of $2 \mathrm{MHz}$. The driving patterns for AM1, AM2 and AM3 as well as the generated replicas are shown schematically in red, purple, blue and orange, respectively. The corresponding pulsewidths and periods are also labelled on the figure. The vertical black dashed line separates the two consecutive frames. c The experimental electrical driving patterns for the three AMs, using same colours as $\mathbf{b}$. In practice, AM2 is only opened for nine times in each frame, since half of the original SUT that directly passes the buffer without being circulated is also considered as one of the ten replicas. d Optical waveform (black trace) of pump and SUT combined together when the system is operated according to the timing chart in $\mathbf{b}$. The pumps scan through the SUT replicas at a step of 150 ps, thus realizing the stroboscopic signal acquisition. Inset, zoom-in of the waveform at the beginning and the end of the scanning. SUT is plotted in orange to be clearly differentiated from the blue pump

will load from input a 5-ns-long waveform as SUT (first horizontal axis). After the SUT is loaded into the buffer, AM2 will be switched on only when the SUT arrives in each circulation. Therefore, in the second horizontal axis, AM2 opens every 40 ns and generates ten identical replicas in each $500 \mathrm{~ns}$ frame. Ideally, the separation of each gating should be identical with the cavity period of the buffer ( $39.85 \mathrm{~ns})$. But limited by the sampling speed of AWG $\left(1 \mathrm{Gs} \mathrm{s}^{-1}\right)$, the separation is set as $40 \mathrm{~ns}$. However, since each SUT is only circulated for ten times inside the buffer and the gating width (10 ns) is much broader than the SUT duration, the slight mismatch between the gating period and the cavity period will not influence the performance of the buffer. After the buffering, ten replicas will be generated with a separation equal to the cavity period (fourth horizontal axis). AM3 performs pulsepicking on the MLL to generate a pump for the time magnifier every $40 \mathrm{~ns}$ (third horizontal axis). The corresponding real electrical driving signals for three AMs are shown in Fig. 4c. Owing to the period difference ( $150 \mathrm{ps})$ between the time magnifier and the SUT replicas, the time magnifier will scan the SUT from left to right with a step of $150 \mathrm{ps}$, thus realizing the stroboscopic signal acquisition.

To directly visualize the stroboscopic signal acquisition, amplified spontaneous emission from an erbium-doped fibre amplifier is used as SUT and combined with time-magnifier pump when the whole system is operated according to the timing chart. The corresponding waveform is shown in Fig. 4d. As observed in the left inset, in each 500 ns period, the first replica of the waveform section (broad and flat pedestal, orange) is aligned with the pump (sharp peak, blue) on the left side while in the last frame, the time-magnifier pump is already scanned to the right side, as shown in the right inset. In this way, the ten output frames will be generated in each $500 \mathrm{~ns}$ period, which corresponds to the magnified waveform at ten consecutive positions of the SUT. By identifying the overlapping areas of the output waveform in neighbouring frames, the ten sections of magnified waveform can be stitched together to reconstruct a much longer continuous waveform (see Supplementary Note 3 for details). Consequently, the recording length of the time magnifier can be scaled by the number of replicas while maintaining the high temporal resolution. Overall, the current PARTI system demonstrates a TBWP of more than 2000, about five times larger than the recording value achieved to date in temporal imaging systems ${ }^{26}$.

Measurement of dissipative Kerr soliton dynamics. Finally, to demonstrate the capabilities of the PARTI system, the system is applied to observe the dynamic evolution of dissipative Kerr solitons inside an ultrahigh- $Q$ microresonator. The corresponding FWM spectrum measured after the time lens is shown in Supplementary Fig. 3. The final output of the system is detected by an $18 \mathrm{GHz}$ photodetector and then digitized and recorded by a real-time oscilloscope. After data processing (see Supplementary Note 3 for details) on the measurement results, two sections of 1.5-ns-long waveform with a 740 fs resolution are reconstructed, 
a

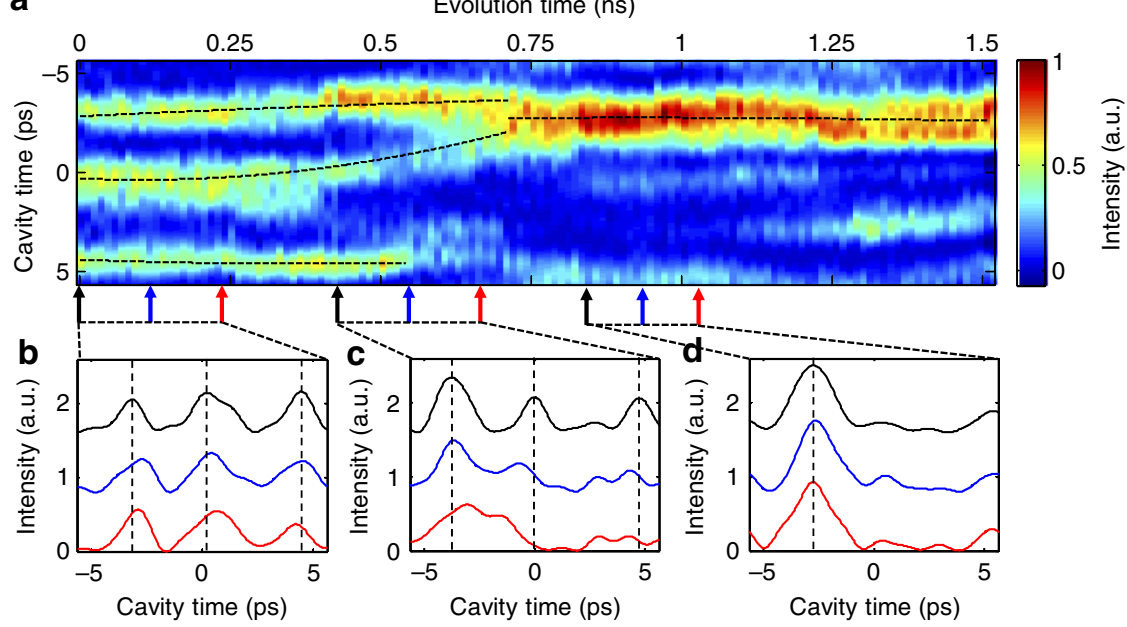

e

Evolution time (ns)

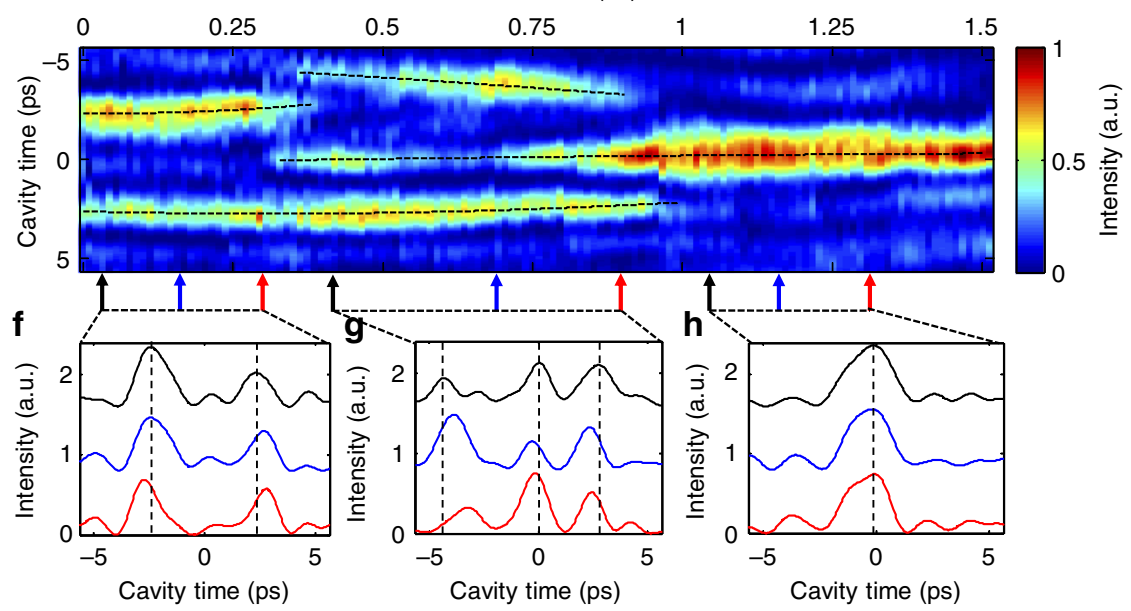

Fig. 5 Dissipative Kerr soliton dynamics measured by the PARTI system. a An example 2D evolution portrait, depicting soliton fusion dynamics and transition from a triplet soliton state to a singlet soliton state. b-d Measured waveforms at different evolution time slices in each stage, illustrating stable triplet solitons at the beginning stage (b), soliton fusion at the middle stage (c) and stable singlet soliton in the final stage (d). e Another example $2 \mathrm{D}$ evolution portrait, showing an evolution from doublet solitons to triplet solitons and eventually to a singlet soliton. $\mathbf{f}-\mathbf{h}$ Measured waveforms at different evolution time slices in each stage, illustrating soliton repulsion at the beginning stage (f), soliton attraction at the middle stage (g) and the stable singlet soliton at the final stage (h). For $\mathbf{a}$ and $\mathbf{e}$, black dashed curves emphasizing the soliton transition traces are plotted against the 2D portrait. For $\mathbf{b}$-d and $\mathbf{f}-\mathbf{h}$ three waveforms at different time slices are plotted in each stage, which are represented in black, blue and red, respectively. Coloured arrows indicate the temporal positions where the waveforms with the corresponding colours are measured. Vertical black dashed lines are plotted according to the soliton positions of the first slice to emphasize the position change of solitons at different time slices

which represent a TBWP of more than 2000. With the unprecedented measurement capability, fascinating dissipative Kerr soliton dynamics in a high- $Q$ microresonator is observed. To clearly visualize the evolution details, we section the onedimensional waveform according to the cavity roundtrip time (11.29 ps) of the microresonator to rearrange the data into a $2 \mathrm{D}$ matrix and create $2 \mathrm{D}$ evolution portraits to depict the dissipative Kerr soliton transition dynamics.

In the first case, a transition process that resembles the simulation result in Fig. 1a is observed. As shown in Fig. 5a, at the beginning stage ( $0 \mathrm{ps}$ to $\sim 400 \mathrm{ps}$ ), three solitons (triplet state) with almost equal intensity exist in the cavity. Figure $5 \mathrm{~b}$ plots the waveforms at three different time slices of 0 ps (black), 113 ps (blue) and $237 \mathrm{ps}$ (red), which shows that the triplet solitons roughly maintain their intensities and positions in the cavity throughout the beginning stage. The three curves were vertically offset for clarity and vertical black dashed lines are plotted according to the soliton positions at $0 \mathrm{ps}$ (black curve) to emphasize the position change of solitons at different time slices.
After that, in the middle stage (400 to $\sim 800 \mathrm{ps}$ ), the first two solitons start to be attracted to each other and eventually merge into a singlet soliton at $\sim 800$ ps. The third soliton is also shifted upwards during the merging of the other two solitons, just like the simulation in Fig. 1a. However, the third soliton does not survive during the transition and starts to fade after 500 ps. The soliton fusion details are shown in Fig. $5 c$, where waveforms at 440, 565 and $677 \mathrm{ps}$ are shown. At these three specific time positions, the separation between the first two solitons evolves from 3.8 to 3 ps and then to 1.5 ps. After this transitioning middle stage, a singlet soliton state is achieved inside the cavity, and the state remains for more than 600 ps, or 53 cavity roundtrips. Similarly, three waveforms in this stage are shown in Fig. 5d, indicating the high stability during the final stage. Black dashed curves emphasizing the soliton transition traces are plotted against the $2 \mathrm{D}$ portrait in Fig. 5a,e, which is obtained by polynomial fitting the peak positions of the solitons.

In addition to the first example, a different dynamic process is also observed which also generates the singlet soliton state 
eventually but without soliton fusion. As shown in Fig. 5e, in the first stage ( 0 to $\sim 370 \mathrm{ps}$ ) two solitons co-exist in the cavity. In the meantime, the doublet solitons repulse each other slightly and the first soliton gradually fades away. At $\sim 370 \mathrm{ps,} \mathrm{the} \mathrm{upper}$ soliton disappears, but at the same time two other solitons emerge. In the second stage (370-1 ns), in contrast to the first stage, the triplet solitons are attracted to the centre slowly. At the end of the second stage, both the top and bottom solitons fade away, while the middle one survives and evolves into a singlet soliton with higher intensity in the final stage (1-1.5 ns). Similar to the first example, the singlet soliton state is much more stable compared to previous states and lasts over $500 \mathrm{~ns}$. Again, waveforms at three different time slices are plotted together for each distinct stage, which shows weak pulse repulsion (f), weak pulse attraction (g) and stable single soliton state (h).

Notably, these two soliton dynamics are observed with the same excitation protocol in the same microresonator. It has been shown, both theoretically and experimentally 29,39 , that the dissipative Kerr soliton formation is not a deterministic process, and soliton states with different orders can be accessed with a certain probability. The reason for such a stochastic behaviour is that these dissipative Kerr solitons can only be generated after the transition from chaos states which by nature are sensitive to initial conditions and noise processes. Future application of PARTI can unveil the probability function and depict the route out of chaos, leading to a better understanding of dissipative Kerr soliton formation. Specific excitation protocols to avoid the chaotic region have been proposed and theoretically studied ${ }^{60}$, and it can be realized and verified experimentally in conjunction with PARTI. Furthermore, PARTI can be applied to study other fascinating nonlinear dynamics including Kerr frequency comb generation beyond Lugiato-Lefever equation ${ }^{61}$ and novel modelocking dynamics via Faraday instability ${ }^{62}$.

\section{Discussion}

As the first proof-of concept demonstration, the stroboscopic signal acquisition (temporal scanning) is performed conservatively to ensure the accuracy of waveform reconstruction. It is worth noticing that while the single-shot recording length of the time magnifier is as large as $300 \mathrm{ps}$, the current temporal scanning adopts a step-size of only $150 \mathrm{ps}$. Therefore, in two consecutive scanning steps, $\sim 50 \%$ of the measurement results are repetitive, which are used as the reference for waveform stitching. Despite this conservative configuration, 1.5-ns-long waveforms are reconstructed with $740 \mathrm{fs}$ resolution, representing a recordhigh TBWP of 2027. The TBWP of the system will be further substantially scaled by reducing the repetitive percentage in neighbouring steps and by increasing the number of buffering times. Currently, the step size is limited by the non-uniform responsivity across the recording window of the parametric time lens (Supplementary Fig. 3d). Because of the worse signal-tonoise near the boundaries of recording window, a small step size is adopted during experiment, so that the reconstructed long waveforms only consist of the high-quality waveforms in the centre area of the recording window. An optical source with higher spectral flatness and nonlinear media with smaller TOD will contribute to a more uniform responsivity across the recording window of the time lens. Ideally, with a flat responsivity, the step-size can be identical with the recording length (i.e., no overlapping areas) to reconstruct continuous dynamic waveforms. Moreover, using a better designed optical buffer, the number of buffered replicas can also be substantially increased (see Supplementary Note 4 for detailed analysis). For example, some optical buffers have demonstrated generating more than 100 replicas with acceptable signal-to-noise degradation ${ }^{63,64}$.
Therefore, under the scenario of non-overlapping scanning and 100 times buffering, the PARTI system can theoretically capture a 30-ns-long non-repetitive dynamic waveform with $740 \mathrm{fs}$ resolution (TBWP larger than $4 \times 10^{4}$ ), which will serve as a powerful tool for studying different kinds of ultrafast optical dynamics. Moreover, the generalized idea of waveform replication combined with single-shot acquisition is also applicable to other measuring techniques, such as the real-time spectral interferometry ${ }^{52}$. Therefore, our technique not only represents an advanced temporal imaging system but may also stimulate more analogous innovations in the family of single-shot ultrafast measurement techniques.

In conclusion, a PARTI system is developed by integrating a fibre-loop-based optical buffer with a low-aberration time magnifier. In analogy to a conventional microscope achieving larger field-of-view by scanning the sample and stitching microscopic images, our technique provides the possibility to observe ns-long dynamic waveforms while maintaining the sub-picosecond temporal resolution, thus overcoming the limitation of TBWP in conventional temporal imaging systems. As a proof-of-concept demonstration, the PARTI system is applied to observe the dissipative Kerr soliton transition dynamics in an ultrahigh- $Q$ microresonator. By buffering the selected waveform ten times and measuring the waveform in ten steps, 1.5-ns-long evolution processes are reconstructed with 740 fs resolution, which represents a TBWP of over 2000, about five times larger than the record value demonstrated to date in conventional temporal imaging systems ${ }^{26}$. Moreover, the TBWP in our technique is scalable to even higher values by using larger number of replicas. With our technique, two distinct multiplet-to-singlet dissipative Kerr soliton transition dynamics are observed. The capability in observing such intriguing phenomena using long recording length and high resolution will not only facilitate the study of dissipative soliton dynamics but also various ultrafast dynamic processes in other fields as well.

\section{Methods}

Experimental setup. The optical buffer consisted of a four-port 50/50 coupler, an optical delay line, an amplitude modulator, a WDM filter, a PC, a 980/1550 WDM coupler, $0.5 \mathrm{~m}$ DCF (DCF38) and $2 \mathrm{~m}$ EDF (ER30-4/125). The net dispersion was estimated by measuring the pulse-broadening of a femtosecond source with an intensity auto-correlator after single-passing the open-loop buffer. The cavity period of the buffer was measured by buffering a picosecond pulse and measuring the separation of buffered replicas. The pump of the temporal magnification system was generated from a $250 \mathrm{MHz}$ optical frequency-comb source (Menlo FC1500250-WG), which was bandpass-filtered (1554-1563 nm) and pulse-picked by AM3 to $25 \mathrm{MHz}$. The input dispersion consisted of $200 \mathrm{~m} \mathrm{DCF}$ (LLMicroDK) and 1.486 $\mathrm{km}$ LEAF (Corning), while the pump dispersion consisted of $400 \mathrm{~m} \mathrm{DCF}$ and 2.97 $\mathrm{km}$ LEAF of the same kind. The ratio between the DCF and LEAF was carefully designed to achieve near-zero TOD. The output dispersion was provided by combining two dispersion compensating modules (Lucent DCM) with a total dispersion of $-1.689 \mathrm{~ns} \mathrm{~nm}^{-1}$. The final optical signal was measured by an $18 \mathrm{GHz}$ photodetector (EOT $3500 \mathrm{f}$ ) and subsequently digitized by a $20 \mathrm{GHz}$ real-time oscilloscope (Tektronix MSO $72004 \mathrm{C}$ ) with $100 \mathrm{Gs} \mathrm{s}^{-1}$ sampling rate. The stroboscopic signal acquisition was controlled using a $1.2 \mathrm{GHz}$ photodetector (DET01CFC), an AWG (Tektronix AWG520) and a delay generator (SRS DG645).

$\mathbf{S i}_{\mathbf{3}} \mathbf{N}_{\mathbf{4}}$ microresonator fabrication. First a $5-\mu \mathrm{m}$-thick oxide layer was deposited via plasma-enhanced chemical vapour deposition on p-type 8 " silicon wafers to serve as the under-cladding oxide. Then low-pressure chemical vapour deposition was used to deposit an $800 \mathrm{~nm}$ silicon nitride for the ring resonators, with a gas mixture of $\mathrm{SiH}_{2} \mathrm{Cl}_{2}$ and $\mathrm{NH}_{3}$. The resulting silicon nitride layer was patterned by optimized deep ultraviolet (DUV) lithography and etched down to the buried oxide layer via optimized reactive ion dry etching. Next the silicon nitride ring resonators were over-cladded with a 3 - $\mu \mathrm{m}$-thick oxide layer, deposited initially with lowpressure chemical vapour deposition $(500 \mathrm{~nm})$ and then with plasma-enhanced chemical vapour deposition $(2500 \mathrm{~nm})$. The device used in this study has a ring radius of $250 \mu \mathrm{m}$, a free spectral range of $88.6 \mathrm{GHz}$, and a loaded quality factor $Q$ of $\approx 1,000,000$. 
Dissipative Kerr soliton generation and modelling. The microresonator was pumped by a frequency-tunable continuous-wave laser with an on-chip power of $600 \mathrm{~mW}$. For dissipative Kerr soliton generation, the laser frequency was scanned with a tuning speed of $2 \mathrm{THz} \mathrm{s}^{-1}$, via control of the piezoelectric transducer, across the cavity resonance from the blue side of the resonance. The soliton dynamics was modelled by numerically solving the mean-field Lugiato-Lefever equation with the symmetric split-step Fourier method and the classical Runge-Kutta method. The simulation started from vacuum noise and the temporal resolution was set to $5 \mathrm{fs}$.

Data availability. The data that support the plots within this paper and the findings of this study are available from the corresponding author on request.

Received: 20 December 2016 Accepted: 31 May 2017

Published online: 05 July 2017

\section{References}

1. Dorrer, C. High-speed measurements for optical telecommunication systems. IEEE J. Sel. Topics Quantum Electron. 12, 843-858 (2006).

2. Li, G. Recent advances in coherent optical communication. Adv. Opt. Photon. 1, 279-307 (2009).

3. Kohler, B. et al. Phase and intensity characterization of femtosecond pulses from a chirped-pulse amplifier by frequency-resolved optical gating. Opt. Lett. 20, 483-485 (1995).

4. Dorrer, C. et al. Single-shot real-time characterization of chirped-pulse amplification systems by spectral phase interferometry for direct electric-field reconstruction. Opt. Lett. 24, 1644-1646 (1999).

5. Park, Y., Ahn, T. J. \& Azaña, J. Real-time complex temporal response measurements of ultrahigh-speed optical modulators. Opt. Express 17, 1734-1745 (2009)

6. Fujimoto, J. G. et al. Femtosecond optical ranging in biological systems. Opt Lett. 11, 150-152 (1986).

7. Li, B., Zhang, C., Kang, J., Wei, X., Tan, S. \& Wong, K. K. $109 \mathrm{MHz}$ optical tomography using temporal magnification. Opt. Lett. 40, 2965-2968 (2015).

8. Li, B., Wei, X., Tan, S., Kang, J. \& Wong, K. K. Compact and stable temporally magnified tomography using a phase-locked broadband source. Opt. Lett. 41, 1562-1565 (2016)

9. Herink, G., Jalali, B., Ropers, C. \& Solli, D. R. Resolving the build-up of femtosecond mode-locking with single-shot spectroscopy at $90 \mathrm{MHz}$ frame rate. Nat. Photon. 10, 321-326 (2016).

10. Wei, X., Zhang, C., Li, B. \& Wong, K. K. Y. Observing the spectral dynamics of a mode-locked laser with ultrafast parametric spectro-temporal analyzer. Paper STh3L.4, CLEO 2015, OSA Technical Digest (Optical Society of America, 2015).

11. Cundiff, S. T., Soto-Crespo, J. M. \& Akhmediev, N. Experimental evidence for soliton explosions. Phys. Rev. Lett. 88, 073903 (2002).

12. Runge, A. F., Broderick, N. G. \& Erkintalo, M. Observation of soliton explosions in a passively mode-locked fiber laser. Optica 2, 36-39 (2015).

13. Solli, D. R., Ropers, C., Koonath, P. \& Jalali, B. Optical rogue waves. Nature 450, 1054-1057 (2007).

14. Suret, P. et al. Single-shot observation of optical rogue waves in integrable turbulence using time microscopy. Nat. Commun. 7, 13136 (2016).

15. Närhi, M. et al. Real-time measurements of spontaneous breathers and rogue wave events in optical fibre modulation instability. Nat. Commun. 7, 13675 (2016).

16. Van Kampen, M. et al. All optical probe of coherent spin waves. Phys. Rev. Lett. 88, 227201 (2002).

17. Schoenlein, R. W., Lin, W. Z., Fujimoto, J. G. \& Eesley, G. L. Femtosecond studies of nonequilibrium electronic processes in metals. Phys. Rev. Lett. 58, 1680-1683 (1987).

18. Goda, K., Tsia, K. K. \& Jalali, B. Serial time-encoded amplified imaging for real-time observation of fast dynamic phenomena. Nature. 458, 1145-1149 (2009).

19. Kolner, B. Space-time duality and the theory of temporal imaging. IEEE J. Quantum Electron. 30, 1951-1963 (1994).

20. Bennett, C. V. \& Kolner, B. H. Principles of parametric temporal imaging. I. System configurations. J. Quantum Electron. 36, 430-437 (2000).

21. Salem, R., Foster, M. A. \& Gaeta, A. L. Application of space-time duality to ultrahigh-speed optical signal processing. Adv. Opt. Photon. 5, 274-317 (2013).

22. Bennett, C. V., Scott, R. P. \& Kolner, B. H. Temporal magnification and reversal of $100 \mathrm{~Gb} / \mathrm{s}$ optical data with an up-conversion time microscope. Appl. Phys. Lett. 65, 2513-2515 (1994).

23. Broaddus, D. H. et al. Temporal-imaging system with simple external-clock triggering. Opt. Express. 18, 14262-14269 (2010).
24. Salem, R. et al. High-speed optical sampling using a silicon-chip temporal magnifier. Opt. Express. 17, 4324-4329 (2009).

25. Pasquazi, A. et al. Time-lens measurement of subpicosecond optical pulses in CMOS compatible high-index glass waveguides. IEEE J. Sel. Topics Quantum Electron. 18, 629-636 (2012).

26. Foster, M. A. et al. Silicon-chip-based ultrafast optical oscilloscope. Nature. 456, 81-84 (2008).

27. Zhang, C., Li, B. \& Wong, K. K. Y. Ultrafast spectroscopy based on temporal focusing and its applications. IEEE J. Sel. Topics Quantum Electron 22, 295-306 (2016).

28. Zhang, C., Xu, J., Chui, P. C. \& Wong, K. K. Parametric spectro-temporal analyzer (PASTA) for real-time optical spectrum observation. Sci. Rep. 3, 2064 (2013).

29. Herr, T. et al. Temporal solitons in optical microresonators. Nat. Photon. 8 , 145-152 (2014).

30. Huang, S.-W. et al. Mode-locked ultrashort pulse generation from on-chip normal dispersion microresonators. Phys. Rev. Lett. 114, 053901 (2015).

31. Yi, X., Yang, Q. F., Yang, K. Y., Suh, M. G. \& Vahala, K. Soliton frequency comb at microwave rates in a high-Q silica microresonator. Optica 2, 1078-1085 (2015).

32. Huang, S. W. et al. A low-phase-noise $18 \mathrm{GHz}$ Kerr frequency microcomb phase-locked over 65 THz. Sci. Rep. 5, 13355 (2015).

33. Liang, W. et al. High spectral purity Kerr frequency comb radio frequency photonic oscillator. Nat. Commun. 6, 7957 (2015).

34. Huang, S. W. et al. A broadband chip-scale optical frequency synthesizer at $2.7 \times 10-16$ relative uncertainty. Sci. Adv. 2, e1501489 (2016).

35. Del'Haye, P. et al. Phase-coherent microwave-to-optical link with a selfreferenced microcomb. Nat. Photon. 10, 516-520 (2016).

36. Papp, S. B. et al. Microresonator frequency comb optical clock. Optica 1, 10-14 (2014).

37. Pfeifle, J. et al. Optimally coherent Kerr combs generated with crystalline whispering gallery mode resonators for ultrahigh capacity fiber communications. Phys. Rev. Lett. 114, 093902 (2015).

38. Lamont, M. R., Okawachi, Y. \& Gaeta, A. L. Route to stabilized ultrabroadband microresonator-based frequency combs. Opt. Lett. 38, 3478-3481 (2013).

39. Zhou, H. et al. Stability and intrinsic fluctuations of dissipative cavity solitons in Kerr frequency microcombs. IEEE Photon. J 7, 1-13 (2015).

40. Turitsyna, E. G. et al. The laminar-turbulent transition in a fiber laser. Nat Photon. 7, 783-786 (2013).

41. Turitsyna, E. G., Falkovich, G. E., Mezentsev, V. K. \& Turitsyn, S. K. Optical turbulence and spectral condensate in long-fiber lasers. Phys. Rev. A. 80, 031804R (2009).

42. Grelu, P. \& Akhmediev, N. Dissipative solitons for mode-locked lasers. Nat. Photon. 6, 84-92 (2012)

43. Dudley, J. M., Dias, F., Erkintalo, M. \& Genty, G. Instabilities, breathers and rogue waves in optics. Nat. Photon. 8, 755-764 (2014).

44. Giordmaine, J. A., Duguay, M. A. \& Hansen, J. W. Compression of optical pulses. IEEE J. Quantum Electron QE-4, 252-255 (1968).

45. Kolner, B. H. Active pulse compression using an integrated electro-optic phase modulator. Appl. Phys. Lett. 52, 1122-1124 (1988).

46. Kauffman, M. T., Godil, A. A., Auld, B. A., Banyai, W. C. \& Bloom, D. M. Applications of time lens optical systems. Electron. Lett. 29, 268-269 (1993).

47. Hirooka, T. \& Nakazawa, M. All-optical $40 \mathrm{GHz}$ time-domain Fourier transformation using XPM With a dark parabolic pulse. IEEE Photon. Technol. Lett. 20, 1869-1871 (2008).

48. Ng, T. T. et al. Compensation of linear distortions by using XPM with parabolic pulses as a time lens. IEEE Photon. Technol. Lett. 20, 1097-1099 (2008).

49. Walmsley, I. A. \& Dorrer, C. Characterization of ultrashort electromagnetic pulses. Adv. Opt. Photon. 1, 308-437 (2009).

50. Kane, D. J. \& Trebino, R. Single-shot measurement of the intensity and phase of an arbitrary ultrashort pulse by using frequency-resolved optical gating. Opt. Lett. 18, 823-825 (1993).

51. Fontaine, N. K. et al. Real-time full-field arbitrary optical waveform measurement. Nat. Photon. 4, 248-254 (2010).

52. Asghari, M. H., Park, Y. \& Azaña, J. Complex-field measurement of ultrafast dynamic optical waveforms based on real-time spectral interferometry. Opt. Express 18, 16526-16538 (2010).

53. Ma, B. et al. Use of autostitch for automatic stitching of microscope images. Micron 38, 492-499 (2007).

54. Rankov, V., Locke, R. J., Edens, R. J., Barber, P. R. \& Vojnovic, B. in Biomedical Optics 2005 International Society for Optics and Photonics 190-199 (SPIE, San Jose, CA, USA, 2005).

55. Coen, S., Randle, H. G., Sylvestre, T. \& Erkintalo, M. Modeling of octavespanning Kerr frequency combs using a generalized mean-field Lugiato-Lefever model. Opt. Lett. 38, 37-39 (2013).

56. Chembo, Y. K. \& Menyuk, C. R. Spatiotemporal Lugiato-Lefever formalism for Kerr-comb generation in whispering-gallery-mode resonators. Phys. Rev. A. 87, 053852 (2013). 
57. Plows, G. S. \& Nixon, W. C. Stroboscopic scanning electron microscopy. J. Phys. E Sci. Inst. 1, 595 (1968).

58. Williams, D., Hale, P. \& Remley, K. A. The sampling oscilloscope as a microwave instrument. IEEE Microw. Mag. 8, 59-68 (2007).

59. Bennett, C. V. \& Kolner, B. H. Aberrations in temporal imaging. IEEE J. Quantum Electron. 37, 20-32 (2001).

60. Jaramillo-Villegas, J. A., Xue, X., Wang, P. H., Leaird, D. E. \& Weiner, A. M. Deterministic single soliton generation and compression in microring resonators avoiding the chaotic region. Opt. Express 23, 9618-9626 (2015).

61. Hansson, T. \& Wabnitz, S. Frequency comb generation beyond the Lugiato-Lefever equation: multi-stability and super cavity solitons. J.Opt. Soc. Am. B 32, 1259-1266 (2015).

62. Tarasov, N., Perego, A. M., Churkin, D. V., Staliunas, K. \& Turitsyn, S. K. Mode-locking via dissipative Faraday instability. Nat. Commun. 7, 12441 (2016).

63. Langenhorst, R. et al. Fiber loop optical buffer. J. Lightwave Technol. 14, 324-335 (1996)

64. Jolly, A., Gleyze, J. F. \& Jolly, J. C. Static and synchronized switching noise management of replicated optical pulse trains. Opt. Commun. 264, 89-96 (2006).

\section{Acknowledgements}

We can acknowledge the discussions with Drs Baicheng Yao and Xiaoming Wei. This material is based upon work supported by the Air Force Office of Scientific Research under award number FA9550-15-1-0081, the Office of Naval Research (N00014-14-10041), the National Science Foundation (14-38147 and 15-20952), Research Grants Council of the Hong Kong Special Administrative Region, China (Project Nos HKU 17205215, HKU 17208414 and CityU T42-103/16-N), National Natural Science Foundation of China (N_HKU712/16), Innovation and Technology Fund (GHP/050/14GD) and University Development Fund of HKU.

\section{Author contributions}

B.L. and S.W.H. proposed the technique and designed the experiment. B.L., S.W.H. and Y.L. performed the experiment. B.L. and S.W.H. wrote the manuscript. All authors contributed to the analysis of the data and discussion and revision of the manuscript.

\section{Additional information}

Supplementary Information accompanies this paper at doi:10.1038/s41467-017-00093-7.

Competing interests: The authors declare no competing financial interests.

Reprints and permission information is available online at http://npg.nature.com/ reprintsandpermissions/

Publisher's note: Springer Nature remains neutral with regard to jurisdictional claims in published maps and institutional affiliations.

(i) Open Access This article is licensed under a Creative Commons Attribution 4.0 International License, which permits use, sharing, adaptation, distribution and reproduction in any medium or format, as long as you give appropriate credit to the original author(s) and the source, provide a link to the Creative Commons license, and indicate if changes were made. The images or other third party material in this article are included in the article's Creative Commons license, unless indicated otherwise in a credit line to the material. If material is not included in the article's Creative Commons license and your intended use is not permitted by statutory regulation or exceeds the permitted use, you will need to obtain permission directly from the copyright holder. To view a copy of this license, visit http://creativecommons.org/ licenses/by/4.0/.

(c) The Author(s) 2017 